Accelerator Division

Alternating Gradient Synchrotron Department

BROOKHAVEN NATIONAL LABORATORY

Associated Universities, Inc.

Upton, New York 11973

Accelerator Division

Technical Note

AGS/AD/Tech. Note No. 278

TRAPPING DECELERATED ANTI-PROTONS

A. Hershcovitch and Y.Y. Lee

March 26, 1987

Introduction

There have been thoughts ${ }^{1,2}$ of using the AGS, as well as its Booster, Linac, and RFQ, to decelerate AGS produced $3.5 \mathrm{GeV} / \mathrm{c}$ antiprotons to energies as low as $20 \mathrm{keV}$. - Without cooling, ${ }^{1}$ during each AGS cycle as many as $10^{5} \overline{\mathrm{p}}$ 's can survive this deceleration process. The trapping of these antiprotons in a relatively inexpensive gated electrostatic trap (aka, Penning trap) is under consideration in this note. An examination of the maximum capability of such a trap reveals that its storage capability far exceeds any conceivable $\bar{p}$ supply. Nevertheless, $\vec{p}$ accumulation in such a trap is rapid and with series addition of traps, the total number of stored antiprotons can exceed those of present day storage rings.

\title{
The Trap
}

Gated electrostatic traps have been used in basic plasma physics experiments ${ }^{3}$ to study single species plasmas. Although these traps have been in use for well over 20 years, 4 it was not until some instabilities were understood and suppressed to enable researchers to achieve remarkable densities of both electron beams and electron gases ${ }^{3}$ for periods of $10^{4} \mathrm{sec}$. In a gated electrostatic trap, charged particles are combined radially by a uniform solenoidal magnetic field and axially by two electrodes biased to a voltage which is high enough to repel the stored particles. The bias on the electrodes can be reduced by pulses to enable particles to enter or to exit the trap, hence, the electrodes function as gates. Beams of antiprotons can be trapped using the following processes: before low energy $\bar{p}$ 's exit the RFQ, the voltage on the gate closer to it is lowered to enable entry into the trap. The antiprotons are reflected at the opposite gate, and the potential on the entry gate is raised just as the leading antiprotons return to it. This results in trapped antiprotons with which experiments can be performed. 
In the absence of instabilities under ultra-high vacuum conditions ( $10^{-10}$ Torr or better), the maximum density of trapped particles is determined by equilibrium conditions of such a non-neutral plasma (in such a high vacuum space charge neutralization can be neglected). This equilibrium state can be investigated using the equation of motion of a trapped particle, i.e., the equilibrium of a single charged particle. For an antiproton in such a $\bar{p}$ plasma column to be in equilibrium, the radially outward centrifugal and electric forces acting on this antiproton must be balanced by the radial inward magnetic force. In the case of a uniform axial magnetic field and an axially symmetric electric field (neglecting the small diamagnetic correction due to the the rotation of $\bar{p}$ 's), the equation of motion of a $\bar{p}$ in equilibrium describing a circular orbit is

$$
-\frac{m v_{\theta}^{2}}{r}=-q E(r)-q v_{\theta} B
$$

where $B$ is the magnetic field, $q$ and $m$ designate charge and mass respectively, $v_{\theta}$ is the azimuthal velocity of the antiproton and $E(r)$ is the radial electric field which can be determined from Poisson's equation (in cylindrical coordinates)

$$
\frac{1}{r} \frac{\partial}{\partial r} \mathrm{r} E(r)=-4 \pi q n(r)
$$

If we assume constant density profile, i.e., $n(r) \equiv n$ for $0<r<R$ and $n(r)=0$ for $r>R$, Equation (2) can be integrated to yield

$$
E(r)=\frac{-r 4 \pi q n}{2} \text { for } 0<r<R
$$

or, in terms of the plasma frequency $\omega_{p}^{2} \equiv \frac{4 \pi n q^{2}}{m}$ this equation becomes

$$
E(r)=-\frac{m}{2 q} \omega_{p}^{2} r
$$

Experimental evidence indicates that the density profile in such a trap is "bel1" shaped rather than a square profile. There are functions which are quite suitable to describe such a profile, one of which 
( $J_{o}(r)$ - ordinary Bessel function of the first kind) was used by the author to analyze a gated electron trap. However, since a more accurate analysis introduces only a small numerical correction to Equation (3), which is not important to our analysis, a square density profile is used throughout this note. Introducing the angular velocity $\omega=v_{\theta}^{2} / r$ in Equation (1), substituting for $E(r)$ from Equation (3) into Equation (1), and using the definition of the cyclotron frequency $\Omega \equiv$ $q B / m$, Equation (1) can be written as

$$
-\omega^{2}=\frac{\omega p^{2}}{2}-\omega \Omega
$$

Solving Equation (4) for $w$, in order to find the range of parameters for which equilibrium exists, we obtain

$$
\omega=\frac{\Omega}{2}\left[1 \pm\left(1-\frac{2 \omega^{2}}{\Omega^{2}}\right)^{0.5}\right]
$$

From Equation (5), it becomes obvious that the density limit of a trap is given by the condition

$$
\frac{2 \omega_{\mathrm{p}}^{2}}{\Omega^{2}}=1
$$

In the case of a beam drifting along $B$, this limit is known as Brillouin flow. The plasma (antiproton) column at this density limit is rotating at $\Omega / 2$. Basically, at this limit, the repulsive electrostatic forces (as measured by $\omega_{\mathrm{p}}^{2}$ ) are balanced by the restoring magnetic forces (as measured by ${ }^{2}{ }^{2}$ ).

In a trap with a magnetic field of $10 \mathrm{~T}$, the maximum density of trapped antiprotons that can be stored (using Equation (6)) is $2.63 \mathrm{x}$ $10^{11} \overline{\mathrm{p}} / \mathrm{cm}^{3}$ ! In a 1 -meter 1ong, $1 \mathrm{~cm}^{2}$ cross section, trap, $2.63 \times 10^{13}$ $\overline{\bar{p}}$ 's can be stored. These numbers exceed any conceivable source of antiprotons by orders of magnitude. There are factors that reduce the Iimit set by Equation (6). These are due to instabilities, however, experiments with electrons proved that these instabilities can be stabilized. 3 Also, the electron densities reached in these experiments $^{3}$ were we1l over $10 \%$ of the 1imits set in Equation (6). Since the 
AGS system can produce only $10^{5} \mathrm{p} /$ pulse, we consider a $1 \mathrm{~T}$ trap. Such a trap is inexpensive, and it can store up to $2.6 \times 10^{9} \bar{p} / \mathrm{cm}^{3}$, i.e., a 1 -meter long trap would have stored $10^{6} \overline{\mathrm{p}}$ pulses if they could have been delivered within a storage time. The storage time is determined by collisions with the background gas in absence of instabilities, hence the requirement of an ultra-high vacuum. A $10^{4} \mathrm{sec}$ confinement time was observed at a pressure of $10^{-10}$ Torr for low energy $(\sim \mathrm{eV})$ electrons.

\section{Trap Loading}

A possible way to enhance the target thickness of such a $\bar{p}$ trap is to accumulate as many antiproton pulses as possible, by injecting successively higher energy antiprotons. Consequently, the voltage on the gates needs to be increased accordingly. The number of $\bar{p}$ pulses that can be accumulated is a function of three parameters: (1) $\bar{p}$ confinement time, (2) the increment by which the gate potential is increased, (3) the maximum voltage on the gates.

The $\overline{\mathrm{p}}$ storage time is most probably dominated by scattering due to collisions with background gas molecules rather than annihilation, since calculated annihilation cross sections ${ }^{5}$ decreases very rapidly at energies above $10 \mathrm{eV}$. Elastic scattering cross sections for $\bar{p}-\mathrm{N}_{2}$ collisions for antiprotons with energies of $10^{\prime} \mathrm{s}$ of $\mathrm{keV}^{\prime} \mathrm{s}$ should be similar to those of electrons having the same relative velocity, since $\mathrm{p}-\mathrm{N}_{2}$ collisions at these energies are dominated by charge exchange. Although electron confinement times (half-1ife) approaching $10^{4} \mathrm{sec}$ ( 2.8 hours) have been observed in gated electron traps, ${ }^{3} \overline{\mathrm{p}}$ storage time will be somewhat lower since an antiproton in a trap will spend some time at low velocities at the turning points. This factor may lead to some non-negligible annihilations. Nevertheless, it is reasonable to expect a $\bar{p}$ storage time of about two hours.

Incremental increases of the gate voltages should be much larger than the energy spread of the antiprotons exiting the RFQ. Since the $\overline{\mathrm{p}}$ energy spread is expected to be rather small $(1 \% \lesssim)$, a $5 \%$ incremental increase in gate potential should suffice. The maximum potential on 
the gates can be made rather high, however, in reasonable cost trap, this potential should not exceed $250 \mathrm{kV}$. Therefore, for an initial gate potential of $21 \mathrm{kV}$ (to trap $20 \mathrm{keV}$ antiprotons), 50 incremental increases of $5 \%$ each will result in a final gate voltage of $240.8 \mathrm{kV}$. Since the AGS cycle is $0.5 \mathrm{~Hz}$, these $50 \overline{\mathrm{p}}$ pulses can be accumulated in $100 \mathrm{sec}$. The accumulation itself is done in a fashion similar to the trapping of the first $\bar{p}$ pulse except that only incremental increases in gate voltages are made. After the first $\bar{p}$ pulse is trapped (20 keV antiprotons in a $21 \mathrm{kV}$ trap), a second $\bar{p}$ pulse with an energy slightly exceeding $21 \mathrm{keV}$ is injected into the trap while the voltage on the entry gate remains at $21 \mathrm{kV}$. The voltage on the other gate is raised by $5 \%$ before this pulse (to $22.05 \mathrm{kV}$ ) to repel these antiprotons. Next, the voltage on the entry gate is raised also to $22.05 \mathrm{kV}$ just as the leading antiprotons from the second pulse read it. This process is repeated for 50 pulses. Therefore, as many as $5 \times 10^{6}$ antiprotons can be accumulated in this trap.

\section{Cooling and Stacking}

If one cools ${ }^{1}$ antiprotons in the booster, $10^{8} \overline{\mathrm{p}} / \mathrm{pulse}$ can be injected into such a trap. Hence, in 50 pulses up to $5 \times 10^{9}$ antiprotons can be accumulated in one trap. Since the accumulation time (100 sec) is much shorter than the storage time ( 2 hours), many traps can be filled up depending on the needed duty factor. The traps are to be stacked in series and loaded up sequentially starting with the trap furthest from the RFQ. Once the desired number of filled traps is reached, the potential on all the intermediate gates can be removed and the antiprotons can be "squeezed" into a single shorter trap.

For a 50\% duty factor, i.e., one hour each for $\bar{p}$ accumulation and for their availability for experiments, $1800 \overline{\mathrm{p}}$ pulses can be trapped (50 pulses in each of 36 traps). Thus, a total of $1.8 \times 10^{11}$ antiprotons can be accumulated in one hour. 


\section{References}

1. Y.Y. Lee, AGS/AD Technical Note No. 266 (1986).

2. Y.Y. Lee and D.I. Lowenstein, AGS/AD Technical Note No. 269 (1986).

3. A. Hershcovitch and P. Politzer, Phys. Rev. Lett. 36 (1976) 1365;

A. Hershcovitch and P. Politzer, Phys. Fluids 22 (1979) 249; W. White, J. Malmberg and C. Driscoll, Phys. Rev. Lett. 49 (1982)

1822; C. Driscoll and J. Malmberg, Phys. Rev. Lett 50 (1983) 167.

4. W. Knauer, J. App1. Phys. 37, 602 (1966).

5. J.S. Cohen, R.C. Martin, and W.R. Wadt, Phys. Rev. A, 27, 1821 (1983).

mvh

$\mathrm{ADY} / \mathrm{TECH}$ 